\title{
La délicate gestion des «U-Boot-Bunker » à Bordeaux et Saint-Nazaire, de la Libération aux années 2000
}

The difficult management of the U-Boot-Bunker in Bordeaux and Saint-Nazaire, from the Liberation to the twenty-first century

Jean-Baptiste Blain

\section{OpenEdition \\ Journals}

Édition électronique

URL : http://journals.openedition.org/abpo/3148

DOI : $10.4000 / a b p o .3148$

ISBN : 978-2-7535-4882-4

ISSN : 2108-6443

Éditeur

Presses universitaires de Rennes

Édition imprimée

Date de publication : 15 décembre 2015

Pagination : 121-142

ISBN : 978-2-7535-4880-0

ISSN : 0399-0826

Référence électronique

Jean-Baptiste Blain, «La délicate gestion des « U-Boot-Bunker » à Bordeaux et Saint-Nazaire, de la Libération aux années 2000 », Annales de Bretagne et des Pays de l'Ouest [En ligne], 122-4 | 2015, mis en ligne le 15 décembre 2017, consulté le 19 avril 2019. URL : http://journals.openedition.org/abpo/3148 ; DOI : 10.4000/abpo.3148 


\title{
La délicate gestion des U-Boot-Bunker à Bordeaux et Saint-Nazaire, de la Libération aux années 2000
}

\author{
Jean-Baptiste BLAIN \\ Doctorant en histoire contemporaine - université de La Rochelle, \\ Centre de Recherche en Histoire Internationale et Atlantique (CRHIA)
}

Les politiques menées par les villes portuaires conduisent à de perpétuelles mutations et provoquent, parfois bien involontairement, un regain d'intérêt pour l'histoire de leur patrimoine militaire. L'exemple des anciennes bases allemandes pour sous-marins situées sur la côte atlantique française - les U-Boot-Bunker ${ }^{1}$ - pose la question des logiques de continuité et de rupture qui sous-tendent les politiques de gestion de ces espaces depuis la Libération. Les deux cas de Saint-Nazaire et de Bordeaux seront traités dans cet article. Les trois autres villes de l'arc Atlantique disposant d'U-bunker - Brest, Lorient et La Rochelle - seront simplement évoquées à titre comparatif. Les U-bunkers encore présents dans l'espace urbain de l'Allemagne - l'U-Boot-Montage im Bunker Valentin et l'U-Bunker Hornisse à

1. Différentes tournures orthographiques apparaissent pour qualifier les «bases pour sous-marins allemands ". L'auteur Sönke NEITZEL qui fait figure de référence dans l'historiographie des U-bunkers, par sa publication en 1991 d'une étude précise sur le sujet, propose d'attacher les trois termes Uboot contraction de Unterseeboot - " sous-marin " en français - et bunker pour évoquer un abri fortifié. SöNKE, Neitzel, Die deutschen Ubootbunker und Bunkerwerften - Bau, Verwendung und Bedeutung verbunkerter Ubootstützpunkte in beiden Weltkriegen, Koblenz, Bernard \& Graefe Verlag, 1991. L'ouvrage publié par le Mémorial de Kiel utilise une autre graphie : Rönnau, Jens, Stolperstein der Geschichte: Die Ruine des Kieler U-BootBunkers als Mahnmal und Herausforderung - Kunst, Geschichte, Meinungsstreit, Kiel, Verein Mahnmal Kilian e.V, 1997. Les articles de la presse allemande et les études locales allemandes plus récentes, utilisent également différents vocables. Inge Cavallie enseignante en langue allemande à l'université Montaigne (ex-Bordeaux 3), invite à utiliser la forme suivante : U-Boot-Bunker. C'est ce terme qui sera utilisé dans cet article. En français l'usage de l'appellation courante erronée base sous-marine avec ou sans majuscules, sera utilisé uniquement pour faire référence à la structure culturelle installée dans l'U-Boot-Bunker de Bordeaux. L'usage des termes U-bunker, base d'U-boote (sans " s " à boote puisque le " e " allemand est le marqueur du pluriel), bases de/pour sous-marins/submersibles, abris pour sous-marins seront employés indifféremment. 
Brême -, de la Norvège - Bruno à Bergen, Dora I \& II à Trondheim - et du sud de la France - Martha à Marseille, ne seront par contre pas évoqués.

Si certaines idées relatives à la mise en valeur culturelle et économique des anciennes bases semblent être reprises d'une ville à l'autre, en dépit de méthodes similaires, les pouvoirs locaux n'ont pas une gestion semblable des U-Boot-Bunker. Pour Saint-Nazaire l'objectif, à la fin des années 1980, était de réintégrer l'U-Bunker dans la ville. L'enjeu était à la fois urbanistique - inscrit dans une démarche de " couture urbaine " - et historique, visant à établir une continuité entre passé et présent, la ville tentant ainsi de se réapproprier " son " port. Le projet "Ville-Port ", décliné en trois volets successifs, se concrétise dès le début du Xxi ${ }^{\mathrm{e}}$ siècle. Et pourtant, en mars 2011, deux grues roulantes sont détruites sur le quai du commerce, quai sur lequel est situé l'U-bunker face au « Bassin de Saint-Nazaire ${ }^{2}$ ». Ces grues avaient pourtant toute leur place sur cette scène portuaire remise en valeur par la ville depuis le début des années 1990. À l'initiative de l'association nazairienne "Les 7 soleils " la reproduction d'une vignette géante extraite d'un album d'Hergé, Les 7 Boules de Cristal, est apposée sur le quai du commerce en juin 2004. Elle représente l'une de ces grues soulevant le Capitaine Haddock assis et vociférant sur un paquetage. Cette vignette paraît désormais être la seule représentation d'un matériel portuaire qui n'est aujourd'hui plus visible.

À Bordeaux, pour transformer l'ancien quartier portuaire de Bacalan en zone d'habitations densifiées, la ville tient compte tant bien que mal des contraintes patrimoniales et des sensibilités mémorielles que ce quartier inspire. Mais la municipalité et la communauté urbaine de Bordeaux (CUB) s'interrogent encore sur l'intégration de l'ancienne base d'U-Boote. Celle-ci étant un lieu majeur, tant par sa situation géographique que par sa morphologie, a été affectée à une mission culturelle : la base montre ainsi son histoire au travers de son architecture, tout en servant la culture par sa transformation en musée ${ }^{3}$. De nombreux projets avaient été proposés depuis les années 1980 pour la réutilisation de cette ancienne base de réparation de submersibles allemands et, dès la fin des années 1940, les pouvoirs publics avaient permis à différentes entreprises de réutiliser ces anciens bâtiments militaires construits durant l'Occupation. Face à cette situation alternant entre espace oublié et tantôt espace source de projets de réaménagement, il convient de se demander si les lieux mémoriels que constituent les anciens U-bunkers allemands n'auraient finalement pas été voués à passer d'abord par l'état de friches portuaires pour se métamorphoser ensuite en espaces de loisirs culturels. En d'autres termes, leur réhabilitation actuelle n'est-elle que le moyen d'occuper l'espace rendu disponible par le déplacement, voire l'abandon d'activités industrielles liées au monde portuaire?

2. Il s'agit du bassin à flot $\mathrm{n}^{\circ} 1$.

3. L'U-Boot-Bunker de Bordeaux est en partie utilisé comme musée pour des expositions temporaires. 
Afin de revenir sur l'évolution des U-bunkers de Bordeaux et SaintNazaire, cet article suivra la chronologie des deux villes portuaires depuis la Libération en trois temps. Au cours de l'année 1945, les décisions prises par les pouvoirs politiques au sujet de la conservation des U-bunkers font qu'aucune destruction n'est réalisée. Ensuite, sur une période allant de 1948 à la fin des années 1980, les U-Boot-Bunker deviennent des édifices utiles à la reprise économique. Enfin, de 1990 à la première décennie des années 2000, les « U-Bunkers industriels " deviennent des lieux culturels.

\section{Le choix de la conservation des U-bunkers par les villes portuaires françaises}

Il est habituellement admis que la période de l'immédiat après-guerre n'est pas celle de la mise en valeur du patrimoine. Elle est plutôt considérée comme celle de son élimination à la faveur des destructions de la guerre. La reconstruction des villes nécessitant l'aplanissement des terrains et donc l'arasement des ruines résiduelles, les blockhaus situés dans ces périmètres sont souvent détruits. La destruction du bunker de la Place Gambetta, des abris bétonnés de la place des Quinconces et du cours des Chartrons, du bunker à torpilles et de l'écluse-bunker sur le Quai du Maroc ${ }^{4}$, sont des exemples bordelais parmi tant d'autres. La destruction de bunkers peut aussi être faite par des personnes agissant par " vengeance ${ }^{5}$ ", Paul Virilio écrivait à ce sujet :

"Beaucoup de riverains me déclaraient que ces bornes de béton leur faisaient peur et qu'elles leur rappelaient de trop mauvais souvenirs, bien des fantasmes aussi, car la réalité de l'Occupation ou du nazisme était ailleurs, le plus souvent dans les banals bâtiments administratifs où logeait la Gestapo; mais c'étaient les blockhaus qui symbolisaient la soldatesque ${ }^{6}$."

Cette citation rapportant le ressenti de personnes vis-à-vis des représentations matérielles de l'Occupation pourrait très bien être complétée par la démonstration de l'historien local nazairien Fernand Gueriff ${ }^{7}$ au sujet des

4. Les destructions faites à la hâte et sans anticipation sur d'éventuels travaux futurs, posent de nombreux problèmes de nos jours. Cf. Blain, Jean-Baptiste, Note historique au GPMB concernant les contraintes de faisabilité du réseau de chaleur et de froid pour le CCTV, 4 octobre 2012, 3 p. La Direction de la stratégie et du développement du port de Bordeaux avait soumis une question émanant de la Direction de l'urbanisme de la CUB entité correspondant à la métropole locale), au sujet d'éventuels résidus de blocs de béton provenant de la destruction de l'écluse-bunker de 1947. Il avait été rappelé que ces résidus avaient déjà retardé l'extension de la ligne de tramway B vers Bacalan en 2004, il semblait donc plausible que ces gravats puissent gêner la nouvelle implantation d'un réseau moderne souterrain de tuyauteries.

5. Virilio, Paul, Bunker Archéologie, Paris, Galilée, 2008, p. 19. Voir aussi, l'article de DEGRÉMONT Isabelle, et TRAN, Thao, «Les blockhaus : lieux de conflits, patrimoine de l'oubli? L'exemple du littoral aquitain ", p. 73-88, dans Meynen, Nicolas (dir.), Valoriser les patrimoines militaires - théories et actions, Rennes, PUR, 2010.

6. VIRILIO, Paul, Bunker Archéologie, op. cit.

7. GUÉRIFF, Fernand, Historique de Saint-Nazaire, De la construction du port à nos jours, T2, Guérande, Imprimerie de la Presqu'île, 1963, p. 163. 
arasements de biens civils publics et privés. Fernand Guerrif montre en effet que tous les prétextes furent bons pour détruire certains immeubles abîmés par les bombardements plutôt que de les réhabiliter ${ }^{8}$ :

«[P]lus de 120 entreprises, venues de tous les horizons, s'étaient précipitées comme des oiseaux de proie, sur les chantiers de démolition. Les Ponts et Chaussées se trouvèrent débordés dans leur surveillance. Les démolisseurs patentés en profitèrent pour prendre des "initiatives hardies". [...] Le scandale des "ronds-jaunes" : ces ronds jaunes signalaient les maisons susceptibles d'être réparées. Pas d'histoire! on démolissait, on rasait sans raison et à tour de bras!»

Il apparaît cependant que les deux exemples sont de nature différente. Les destructions des domiciles privés nazairiens n'ont pas pour but de faire table rase de l'habitat d'avant-guerre, la destruction a seulement un intérêt financier pour les démolisseurs. Si la période de la Reconstruction ne semble pas être celle de la protection du patrimoine au sens général, les bunkers édifiés en France paraissent pourtant faire figure d'exception. Les $U$-Boot-Bunker construits en Norvège ${ }^{9}$ à Trondheim (Dora I et Dora II), Bergen (Bruno) et en Allemagne à Kiel (Kilian et Konrad), Helgoland (Nordsee III), Hamburg (Elbe II et Fink II) et Brême (Hornisse et Valentin), subirent tous des tentatives de destructions après la guerre ${ }^{10}$. Ces dernières furent réalisées dans le cadre de la démilitarisation de l'ancien Reich nazi par les Britanniques à partir de mai 1945. Sur les huit U-bunkers cités, cinq furent complètement détruits dans l'immédiat après-guerre. Le cas de l'île d'Helgoland est emblématique de ces destructions : l'immense cratère visible sur des clichés aériens " est la conséquence de la plus grande explosion non-nucléaire jamais enregistrée au cours de l'Histoire. Le RoyaumeUni fit exploser plus de 6700 tonnes de TNT $^{11}$ dans les installations militaires allemandes, détruisant une grande partie de la zone Sud de l'île ${ }^{12}$ ". En France, contrairement à l'Allemagne et à la Norvège, aucune alvéole-bunker n'a été détruite après la guerre par les Alliés.

L'ancienne base de sous-marins de Saint-Nazaire est récupérée par la Marine Nationale dès la reddition de la poche allemande le 11 mai $1945^{13}$. Alors que la Libération de la France était en cours, l'armée française possé-

\footnotetext{
8. Ibidem, p. 163-164.

9. Le choix d'inventorier les U-Boot-Bunker, suit un ordre géographique allant du Nord au Sud de l'Europe.

10. Certains de ces U-bunkers n'étaient pas terminés. Ils n'ont donc jamais pu être utilisés aux fins prévues par l'Allemagne nazie et/ou n'ont été " que " partiellement détruits par les Alliés.

11. Dans le cadre de l'Opération Big-Bang le 18 avril 1947, cf. RANSTED, Chris, "Heligoland ", dans ATB (After The Battle), n 154, London, 2001, 34 p.

12. Lalanne, Bernard, MaIRE, Richard, Meyer, Anne-Marie et Prat, Marie-Claire, Les risques et les catastrophes d'origine humaine, Bordeaux, "Les petits vocabulaires de la Géographie ", Confluences, mai 2013, p. 40 notice : " explosion ".

13. SiCARD, Daniel et al. (dir.), La Poche de Saint-Nazaire, Nantes, Siloë, 2005, p. 95. NicoL, Charles, Saint-Nazaire ville maritime et portuaire, Nantes, Siloë, 2004, p. 37.
} 
dait déjà une excellente connaissance de la structure des U-bunkers ${ }^{14}$. Cela explique pourquoi la Marine n'a pas tardé à intervenir auprès des pouvoirs locaux à propos de la gestion de ces lieux. Au moment de la Libération, l'intérieur des alvéoles de la base est intact. L'U-510 15 , présent dans l'alvéole $\mathrm{n}^{\circ} 4$, et l'ensemble du matériel provenant des installations électriques de l'U-bunker sont transférés à la base de sous-marins de Brest. Alors que la ville connaît une restructuration, un agent municipal propose en 1948 de faire de l'U-bunker une gare maritime transatlantique ${ }^{16}$ " connectée à une liaison ferroviaire $^{17}$ ". Il est vrai, qu'avant-guerre, le quai où se situe l'U-Boot-Bunker, était celui d'où partaient les navires transatlantiques. Cette première proposition - transformer le lieu militaire en un espace civil - symbolise bien le lien entre le passé et le présent. En effet " le quai de la base de sous-marins " a toujours été un lieu de départs et d'arrivées de paquebots, du XIX siècle jusqu'au début de la Seconde Guerre mondiale. Cet emplacement était d'ailleurs nommé par les Nazairiens "le quai de la Transat' ", alors que son nom officiel était " quai Henri Chevreau ". Celui-ci s'est vu modifié par l'installation des 6 \& 7 U-flottille, puis à partir du mois de février 1941 avec le début de la construction de l'U-bunker. Si la continuité historique du projet de gare maritime semble claire, il ne s'est pourtant jamais réalisé. Peut-être lui manquait-il une vision plus globale dans le domaine des transports? En effet les progrès techniques de l'aviation militaire ont été utilisés dans la branche civile de ce secteur et la " navigation aérienne " a très rapidement supplanté les lignes maritimes. L'U-bunker de Saint-Nazaire, transformé en gare-maritime, n'aurait donc pas pu servir sur le long terme à la seule activité de transport des personnes par voie océanique. Par ailleurs, des réflexions autour d'une éventuelle destruction de l'U-Boot-Bunker de Saint-Nazaire furent conduites, mais jamais concrétisées ${ }^{18}$. Bien que la reconstruction de la ville de Saint-Nazaire ait nécessité la destruction de bâtiments endommagés ou ne pouvant être inclus dans le plan d'aménagement fonctionnaliste de Noël Le Maresquier et de son adjoint André Guillou, finalisé en $1947^{19}$, la reconstruction identitaire n'a cependant pas rendu indispensable la démolition du lieu représen-

14. Cf. note de renseignement $\mathrm{n}^{\circ} 1$, ministère de la Marine, État-major général $2^{\mathrm{e}}$ bureau, $1^{\mathrm{er}}$ mars 1945, 7P153. SHAT Vincennes.

15. D’après Neitzel, Sönke, op. cit., p. 180 et Buffetaut, Yves, " La base de Saint-Nazaire et ses flottilles ", Marines Magazine (M. M.), n 30, Rennes, janvier 2003, p. 68.

16. Cet homme s'appellerait Georges Geffray, il a réalisé une aquarelle présentant son projet, $c f$. SICARD, Daniel, " La Base sous-marine de Saint-Nazaire ", 303 Arts, Recherches et Créations, $\mathrm{n}^{\circ}$ 65, Nantes, $2^{\mathrm{e}}$ trimestre 2000, p. 14. Avant guerre "l'ancienne gare ", dont la façade constitue désormais "le Théâtre " scène nationale de la ville, donnait sur le quai Henri Chevreau soit " le quai de la Transat' ".

17. Batteux, Joël et Guetny, Noël, Vouloir sa ville, Paris, Le Cherche-Midi, 2012, p. 213.

18. Encore récemment fin 2013, une liste d'opposition municipale conduite par un dissident socialiste proposait dans le journal Ouest-France de " raser les deux tiers de la base sous-marine " et sur son site internet de conserver uniquement les seules alvéoles utilisées par le musée sur les paquebots Escal'Atlantic. Cf. LuCAS Amélie, " "Saint-Nazaire revitalisée" vise entre 20 et 25 \% ", Ouest-France, 20 novembre 2013.

19. LE GuEN, Gilbert, "Aspects économiques et urbains de la reconstruction de SaintNazaire ", Annales de Bretagne, tome 60, n² 2, 1953, p. 278. Noël Le Maresquier avait été 
tant l'Occupation allemande, l'U-bunker. En effet, de 1940 à 1944 et durant la période de "la poche ", les Nazairiens ont su se réapproprier leur rôle de citoyens français en participant à plusieurs formes de résistance contre les Allemands ${ }^{20}$. La poche de Saint-Nazaire peut être considérée comme un "sas mental » pour la population. La base de sous-marins à la Libération n'est donc pas uniquement considérée négativement, elle est aussi vue comme un bâtiment aux multiples fonctionnalités. Une étude portant sur son éventuelle destruction est néanmoins conduite dès 1945 par les pouvoirs publics civils, mais " le coût élevé de l'opération (478 millions de francs de l'époque) et les dangers des explosions dans un rayon de 400 mètres arrêtent le projet des destructeurs ${ }^{21}$ ". "Les destructeurs ", comme ils sont nommés par Daniel Sicard, conservateur de l'Écomusée de Saint-Nazaire de janvier 1991 à septembre 2013, sont les personnels du service de l'urbanisme et de l'habitation et plus précisément le commissaire au remembrement de Saint-Nazaire. Celui-ci, sollicitant trois ans plus tard le préfet de la Loire-Inférieure de lui accorder explicitement l'autorisation de détruire l'U-bunker, se voit attribuer une fin de non-recevoir. Il est au contraire stipulé dans la lettre que le préfet adresse au service de l'urbanisme et de l'habitation le 27 janvier 1948 que le bâtiment doit demeurer en parfait état de fonctionnement :

"Par lettre citée en référence vous m'avez prié de vous faire connaître si une décision définitive avait été prise par les autorités compétentes pour la conservation ou de la démolition de la base sous-marine de St-Nazaire. $\mathrm{Au}$ reçu de votre lettre, je me suis mis en rapport avec M. le Contre-Amiral, Commandant l'arrondissement maritime de LORIENT qui m'a transmis en réponse copie des D.M. 1922 T.M 2 du $1^{\text {er }}$ décembre 1947 de la Direction Centrale des Travaux Immobiliers et Maritimes et de la D.M. 13 C. 168 A du 16 décembre 1947 du Ministère des Travaux Publics et des Transports. De ces décisions ministérielles, il ressort que le Ministère des Forces Armées (Marine) n'envisage nullement la démolition de la base sous-marine de St-Nazaire. Une commission mixte - service maritime des Ponts et Chaussées de la Loire Inférieure et Direction des Travaux Maritimes de LORIENT - étudie le partage de la base entre le secteur civil et la marine et doit définir les conditions d'entretien pour maintenir en permanence, en bon état de marche, les installations de la partie non conservée par la marine ${ }^{22}$."

nommé architecte en chef de la reconstruction de Saint-Nazaire dès 1943 par le régime de Vichy et fut confirmé dans ses fonctions à la Libération.

20. L'opération commando "Chariot " des forces alliées du 28 mars 1942 avait déjà révélé que la résistance bretonne était présente à Saint-Nazaire. Parmi les nombreuses publications traitant de "l'Opération Chariot ", il peut être conseillé la lecture de la synthèse de Chemereau, Hubert, "Opération Chariot, Saint-Nazaire dans la bataille de l'Atlantique ", ArMen, n 187, mars-avril 2012, p. 40-47. Plus largement sur les réseaux de la Résistance bretonne voir, SAINCLIVIER, Jacqueline, La Bretagne dans la guerre 1939-1945, Rennes, Ouest-France, 1994, 219 p.

21. SICARD, Daniel, "La base sous-marine... ", op. cit., p. 10.

22. Préfet de la Loire Inférieure à Monsieur le délégué départemental à la Reconstruction et à l'Urbanisme 52, rue de Carcouët Nantes. Objet : Base sous-marine de Saint-Nazaire. Ref. V/lettre ML/SP - 68.304 - Service de l'Urbanisme et l'habitation - Section de l'Urbanisme du 27 janvier 1948. Archives dép. Loire-Atlantique dossier 75 W 243, consulté le 14 mars 2014. 
Après avoir envisagé sa destruction trois ans auparavant, un projet de faire de l'ancienne base de sous-marins un lieu touristique ${ }^{23}$ est présenté en 1949. Ce projet n'est pas aussi étrange qu'il y paraît, car à la fin de la Seconde Guerre mondiale éclot une nouvelle forme de tourisme, "le tourisme de ruines ${ }^{24}$ ». Le projet de transformation touristique de la base de sous-marins reste dans les esprits, mais il ne sera réalisé que cinquante et un ans plus tard.

À Bordeaux la décision de détruire la base de sous-marins aurait pu, au contraire, se comprendre. La Libération ne s'est en effet pas déroulée de la même manière qu'à Saint-Nazaire. Les Bordelais n'ont pas bénéficié de ce sas psychologique qu'aurait pu représenter une poche de résistance allemande. La ville de Bordeaux est en effet libérée avec une relative rapidité, par rapport aux villes classées comme Festung. Le 28 août 1944, les citoyens sont passés de l'Occupation nazie à la Libération, négociée par les Alliés sans véritable transition. Il semble donc compréhensible qu'aucun projet de réutilisation globale de la base bordelaise ne soit envisagé dans les premières années de l'après-guerre. Cette base de sous-marins, située sur un quartier détruit ${ }^{25}$, est laissée en l'état par la Marine et par la nouvelle municipalité de Jacques Chaban-Delmas. L'intérieur de ses alvéoles avait subi d'importantes destructions perpétrées par les forces d'occupation avant leur départ :

" À la Libération, les alvéoles de la Base et les bassins à flot sont trouvés obstrués par plusieurs bateaux coulés, les grues sont dynamitées. Bateauxportes et pompes sont presque tous inutilisables, les machines ont été envoyées par le fond. Mais le gros œuvre de la Base est quasi intact, de même que l'écluse couverte et les casemates se trouvant aux environs immédiats ${ }^{26}$. "

À ces destructions s'ajoutent celles de l'arme sous-marine ennemie. Trois submersibles l'U.IT. 21 - ex-Guiseppe Finzi de la BETASOM ${ }^{27}$ (classe Pietro Calvi) ${ }^{28}$-, l'U188 (type IXC 40) ${ }^{29}$ de la 10 U-Flottille de Lorient et l'U178

23. TARDIF, Stéphane, "Une cité se sépare de son port... ", op. cit., p. 38.

24. Elle est évoquée dans la revue Urbanisme $n^{\circ} 114$ d'avril 1947. Les promoteurs nazairiens de cette forme de tourisme espèrent que des vacanciers passent par la ville sinistrée. Paul Virilio commençait lui aussi son ouvrage, Bunker archéologie, op. cit., par sa "découverte de la mer" au cours de l'été 1945 (p. 13).

25. Voir aussi, Krause, Peter, Bordeaux, les bombardements, Montreuil-Bellay, éd. CMD, coll. 1940-1944, 151 p.

26. Francis SALlaberRy, L'Aquitaine Allemande, Biarritz, éd. J\&D, p. 96.

27. "BETASOM " était le nom de code de la base italienne de submersibles opérationnelle de septembre 1940 à septembre 1943 sur le Bassin à flot $n^{\circ} 1$ de Bordeaux. BETA fait référence à la lettre grecque " $\beta$ - B " et " som " étant le diminutif de " sommergibile/i * submersible(s) en italien.

28. Ce sommergibile est sabordé au Verdon. Cf. ADMIRALTY, German, Italian and Japanese U-Boat Casualties during the War-Particulars of destruction, His Majesty's Stationery Office, London June 1946, p. 20 : "Kill no 568 - 25.8.44 - IT.21Destroyed - "S" Other Causes Bordeaux (Biscay)", cette indication est confirmée par les références suivantes : NAVAL History Division Office of the Chief of NAval Operations Washington, United States submarine losses World war II, reissued with an appendix of Axis submarine losses, 1963.

29. NeITZEL, Sönke, Die deutschen Ubootbunker..., op. cit., p. 184. 
(type IX D2) ${ }^{30}$ de la 12 U-Flottille de Bordeaux, sont sabordés avant l'arrivée des Libérateurs ${ }^{31}$. Des débris d'explosion projetés sur la toiture intérieure de l'alvéole $\mathrm{n}^{\circ} 8$, provenant soit d'un matériel portuaire soit d'un bateau, sont encore visibles de nos jours. S'ajoutent aux destructions le blocage de l'embouchure de la Gironde, plus communément appelé le barrage de Lagrange. Le port est ainsi rendu inaccessible pendant une bonne année. La base de sous-marins ne semble donc pas présenter un autre intérêt que celui de l'appontement de navires, tels les premiers Liberty-ships qui parviennent au port de la Lune à partir de 1945. Par ailleurs, la localisation du quartier de Bacalan situé au nord de Bordeaux, dans une zone industrielle longtemps considérée comme " une fin de ville ", n'est pas un espace visible à partir du centre-ville. Seules les personnes travaillant sur la zone des bassins à flot et les Bacalanais ${ }^{32}$ cohabitent avec l'U-bunker. C'est pourquoi une destruction de l'U-bunker pour embellir la ville ne semble pas avoir été proposée ${ }^{33}$.

La présence actuelle des abris pour submersibles sur la côte atlantique française en est la preuve : ces bâtiments ne furent jamais détruits par les pouvoirs publics, contrairement à d'autres bunkers de servitude, telle l'écluse fortifiée de Bordeaux arasée en 1947. Ces édifices, du fait de leur gigantisme, servent à partir de la fin des années 1940 à l'installation d'industries. Cette mutation des U-Boot-Bunker nazairiens et bordelais en "bunker-industriels " est permise par la Marine Nationale qui demeure, jusqu'à la fin des années 1980, propriétaire des murs. Ainsi, toute demande de travaux dans les U-Boot-Bunker nécessaires à l'installation d'entreprises civiles, passe par l'Amirauté.

\section{Les U-bunkers, des bâtiments utiles pour la reprise économique de villes françaises}

À Bordeaux, différentes entreprises profitent du gigantesque hangar que constitue la base pour diverses activités de petites et moyennes industries.

"Installations louées. La paix revenue, la marine nationale - chargée de l'utilisation de la base en cas de nouveau conflit ${ }^{34}$ - confia les installa-

30. Ibidem, "Das Boote hatte sich im Bunker mit zerfetztem Bug auf die Seite gelegt ".

31. SALLABERRY, Francis, L'Aquitaine Allemande, op. cit., p. 87 et BufFETAUT, Yves, Les Ports de l'Atlantique : les ports français 1939-1945, Nantes, Marines éd., 2003, p. 84.

32. Les habitants du quartier de Bacalan s'appellent eux-mêmes "les Bacalanais "; le quartier étant considéré par ses derniers comme une ville dans la ville. Karim Messaie, chargé de l'aménagement au Port de Bordeaux et enseignant à l'IUT de Bordeaux Montesquieu, préfère l'expression " phénomène d'insularité " pour qualifier cet espace enclavé. Entouré de deux bassins à flot, d'une route d'entrée de ville menant du périphérique intérieur au centre de la ville, du pont d'Aquitaine et de la Garonne, le quartier semble effectivement être un îlot dans l'immensité de l'agglomération bordelaise.

33. La documentation sur ce sujet est assez lacunaire, que ce soit aux archives municipales ou départementales de Gironde. Les archives du Centre Jean-Moulin sont, quant à elles, en cours de classement pour un temps indéterminé.

34. La fin de la Seconde Guerre mondiale marque l'entrée dans la Guerre Froide. La Marine a très vite intégré cette nouvelle donne géostratégique dans sa stratégie de 
tions au Port Autonome de Bordeaux qui, a son tour, loua les locaux à des entreprises industrielles et commerciales. La réparation navale y occupe plusieurs boxes; l'un d'eux, d'ailleurs, servant à la construction de petits bâtiments ${ }^{35}$."

Sur le fronton de la porte nord de l'U-bunker une inscription à la peinture évoque encore ce passé industriel : "Ateliers Métallurgiques de la Base ${ }^{36}$ ". Mais les informations s'avèrent lacunaires à ce sujet. Peut-être demeure-t-il des cartons non ouverts au Grand port maritime de Bordeaux (GMPB) ou non encore répertoriés aux archives municipales de la ville de Bordeaux.

À Saint-Nazaire, jusqu'en $1955^{37}$, les alvéoles-bunkers servent également d'espace pour réparer et construire de petits bâtiments auxiliaires pour la Marine. Celle-ci, qui conserve la propriété du bâtiment ${ }^{38}$, accorde " le $1^{\mathrm{er}}$ avril 1946 la gestion de la base à l'administration du port de Saint-Nazaire, dépendant du Ministère des Travaux Publics et des Transports $^{39}$ ". Dès le début des années $1950^{40}$, suite à une convention ${ }^{41}$ avec la chambre de commerce, "l'entreprise franco-tunisienne de l'Hyperphosphates RENO ${ }^{42}$ " utilise les alvéoles 10, 11, 12 et 13 de l'U-bunker ${ }^{43}$ " pour une surface de 9750 mètres carrés y compris la demi-mesure des murs de l'ouvrage considéré ". Cette concession pour l'utilisation de quatre alvéoles est prévue pour dix ans, de 1951 à 1961. Celle-ci se voit toutefois modifiée, pour permettre à l'entreprise Reno d'accroître ses acti-

défense. Aussi toutes les conventions d'occupations accordées par la Marine Nationale aux entreprises civiles, sont soumises à une clause d'annulation " en cas de tensions diplomatique, mobilisation ou guerre "; citation extraite du document : Service Maritime, Convention du 22 juin 1950, page 3, article IV, alinéa A, Série 1W - SHD Rochefort.

35. LACROIX, André, "La base sous-marine résistera à l'épreuve du temps... mais ses aménagements intérieurs menacent ruines ", Sud-Ouest, 22 janvier 1965, p. 5. La recherche que nous menons actuellement au sujet de l'U-bunker de La Pallice, présente de fortes similitudes avec des concessions d'alvéoles accordées à des entreprises par le Port et la Marine durant cette même période.

36. Il est à noter qu'à partir de l'installation d'industries dans les anciens U-Boot-Bunker, les documents mentionnent moins le terme " alvéole " et le remplacent par "box ". Le lieu militaire devenant un espace de stockage industriel, les termes décrivant le bâti des U-bunker évoluent vers le domaine civil.

37. BRAEUER, Luc, Guide souvenir - La base sous-marine, op. cit., p. 64.

38. L'U-Boot-Bunker est racheté par la ville de Saint-Nazaire au ministère de la Défense en juillet 1998, cf. Simonot, Béatrice, "À la recherche d'un nouveau monde ", 303 Arts, Recherches et Créations, $\mathrm{n}^{\circ}$ 65, Nantes, p. 19.

39. BRAEUER, Luc, Guide souvenir - La base sous-marine, op. cit.

40. L'entreprise Reno est créée officiellement le $1^{\text {er }}$ février 1950 (Archives municipales de Saint-Nazaire). Toutefois, cette société semble avoir une histoire antérieure à la guerre.

41. Lettre du préfet de la Loire-Inférieure à l'ingénieur en chef des Ponts et Chaussées service maritime de Nantes datée du 12 septembre 1951, Arch. dép. de Loire-Atlantique.

42. Nommée parfois aussi : "Compagnie Nord Africaine de l'hyperphosphate Réno " ou encore dans les articles du Populaire: "Rhéno " (Archives Municipales de Saint-Nazaire).

43. Lettre datée du 12 décembre 1951 du préfet de la Loire-Inférieure à l'ingénieur en chef des Ponts-et-Chaussées, Service Maritime de Nantes, objet : Base sous-marine de Saint Nazaire - approbation d'un contrat d'occupation, réf. vos rapports des 7 et 29 novembre 1951, Arch. dép. de Loire-Atlantique 75 W 243, sous-série 496 [1956] 2. 243. 
vités. L'usine s'étend ainsi de l'alvéole 9 à l'alvéole 13 et elle obtient même, à partir du mois de juillet 1960, l'autorisation de construire un parking à automobiles, "dans la partie Est du redan sud de la BSM [sur] une bande de terrain de $60 \mathrm{~m}^{2} 44$ ". Pour ce faire, la plupart des alvéoles occupées par l'usine se retrouvent asséchées et comblées par des phosphates. Une nouvelle convention est signée en mars 1962, pour une durée limitée à moins de dix années ${ }^{45}$. L'usine est en effet productive, les besoins agricoles du marché commun européen sont importants et l'emploi des phosphates l'est tout autant. L'entreprise Reno est créatrice d'emplois ${ }^{46}$, cependant les conditions de travail laissent à désirer ${ }^{47}$. Mais, comme son prédécesseur $^{48}$, son second directeur devient en 1964 membre de la commission permanente d'enquête de la chambre de commerce et d'industrie ${ }^{49}$; ceci facilite sans aucun doute les activités de l'entreprise. Parallèlement au développement de ces activités industrielles, indispensables à la reprise économique de l'interface portuaire nazairienne et de son arrière-pays, mais également génératrices d'une certaine nocivité qui aurait conduit au développement de maladies respiratoires chez les enfants du quartier du "Petit Maroc ${ }^{50}$ ", Cargill et un chai de vins provenant d'Algérie profitent de l'infrastructure qu'offre l'U-bunker ${ }^{51}$. "La société des Chais Nazairiens " s'installe dans cette ancienne base pour sous-marins allemands dès la fin

44. Décision acceptée par la CCIE et le Port de Saint-Nazaire le 5 juillet 1960, Arch. dép. de Loire-Atlantique $164 \mathrm{~W} 78$.

45. Aussi, un nouveau calcul de la superficie occupée par l'usine est fait. Pour l'année 1961, la surface totale utilisée est d'environ $12000 \mathrm{~m}^{2}$, la redevance s'élève donc pour cette même année à 11271 Nouveaux Francs.

46. Dans un article du Populaire, daté du 3 octobre 1952 (" Dans la base sous-marine. Dotée d'un matériel ultra-moderne, l'usine nazairienne Rheno traite les phosphates en provenance d'Afrique du Nord "), une question portant sur le nombre de personnes travaillant dans l'usine, obtient pour réponse : «Une soixantaine actuellement; c'est-à-dire deux équipes : c'est un travail saisonnier, il nous est arrivé d'avoir une équipe supplémentaire et de travailler 24 heures sur 24 ".

47. Témoignage de Daniel Sicard recueilli par l'auteur en septembre 2011.

48. Le président de l'entreprise Reno, au moment de l'installation de l'usine de traitement des phosphates dans la base de Saint-Nazaire, était " Jean Le Cornec "; le directeur de la filiale nazairienne était " Monsieur Caillard ». Ce dernier est ensuite remplacé - vraisemblablement en 1963 - par " Monsieur Jourdan ». Les prénoms des directeurs ne sont pas mentionnés dans les archives consultées.

49. Saint-Nazaire le 21 décembre 1963, CCIE de Saint-Nazaire, rue Villès-Martin SaintNazaire, Commission Consultative et Commission permanente d'enquête du port de SaintNazaire. Arch. dép. de Loire-Atlantique, dossier 164 W 78.

50. D'après un témoignage de Monsieur Daniel Sicard et un article de l'Éclair, SaintNazaire, "La société Reno répond... ", 4 juin 1976 (archives municipales de Saint-Nazaire).

51. Arch. dép. de Loire-Atlantique, dossier 1899 W 10 (1945-1951). En outre dès 1964 l'entreprise Soja-France s'installe sur l'avenue de la République à Saint-Nazaire pour y " effectuer ses premières activités de négoce ". En 1970 une première usine est construite. Les activités industrielles de cette société nécessitent également l'utilisation de l'intérieur et du terre-plein nord de la " base sous-marine " de Saint-Nazaire. À partir de 1992 cette entreprise crée une raffinerie pour y produire des tourteaux de soja et de l'huile de tournesol. La firme basée à Saint-Nazaire se concentre désormais sur les activités de transformations du tournesol. Sources : site de Cargill France [http://www.cargill.fr/fr/ 
des années 1950 : des cuves à vins auraient été installées dans l'arrièrebox $\mathrm{n}^{\circ} 14$, la démolition du plancher en béton séparant le premier et le second étage de l'arrière-box $\mathrm{n}^{\circ} 14$ aurait également été nécessaire et réalisée à cette fin ${ }^{52}$. L'importation du vin d'Afrique du Nord servait, au cours de ces années, à couper le vin produit en métropole, afin de lui donner un degré d'alcool plus important. Enfin la voie ferrée, dont il demeure des traces au sein de l'U-Boot-Bunker nazairien, est également construite dans ces années ${ }^{53}$. Dans une lettre en date du mois de décembre 1947 et signée par le secrétaire d'État chargé de la Marine, sont décrits les futurs travaux à réaliser dans l'U-bunker. Durant cette première phase de mutation du bâtiment, les rails intérieurs y auraient été posés. Cette mutation peut être qualifiée d'industrielle ${ }^{54}$.

L'entreprise Cargill, occupe toujours les alvéoles 1 à 5 . Construite à la fin des années 1960, l'usine célèbre en 2014 ses cinquante ans d'activités à Saint-Nazaire. Elle sert à fabriquer de l'huile de tournesol et des tourteaux par trituration. Cette activité s'avère nécessaire dans l'Ouest de la France qui, depuis la fin de la Seconde Guerre mondiale, connaît une forte demande en produits oléo-protéagineux afin de satisfaire les besoins de l'élevage. En plus de cette usine nazairienne aux capitaux américains, la maison mère familiale Cargill basée à Minneapolis ouvre à Brest en 1976 un autre site où 700000 tonnes de soja sont transformées par an. Cette success-story ne s'arrête pas là, car la société - détenue en 2014 à 88 \% par la famille Cargill - se diversifie au début des années 1990, "en s'équipant d'une unité de raffinage qui permet la production d'huile brute. [...] En 2007, la montée en puissance se poursuit pour Cargill avec la naissance à Montoir, d'une usine high-tech de transformation de colza (850000 tonnes/

sites/saint-nazaire/index.jsp] (consulté le 12 avril 2014) et témoignage de Marc Henri, ingénieur en agro-alimentaire, collecté en septembre 2011.

52. L'emploi du conditionnel s'explique par le fait que les documents, consultés aux archives départementales de Loire Atlantique au sujet de la société des Chais Nazairiens, ne font état que de projets de travaux. Des indices précis tels des plans consultés auprès de ces mêmes archives, permettent toutefois au chercheur de penser que les réalisations furent effectives. En outre cette société obtient, via la demande de la chambre de commerce, l'accord du Contre-Amiral Poix de Lorient le 26 novembre 1958, pour la démolition du plancher en béton séparant le $1^{\mathrm{er}}$ et le $2^{\text {nd }}$ étage de l'arrière-boxe ${ }^{\circ} 14$ afin de permettre l'installation de ses cuves à vins.

53. Lettre de l'ingénieur d'arrondissement datée du 26 juillet 1958 à l'intention de l'ingénieur en chef de Saint-Nazaire : "y compris les deux voies ferrées construites par la Société RENO ", Arch. dép. de Loire-Atlantique.

54. Lettre du secrétaire d'État chargé la Marine auprès du ministère des Forces Armées au ministre des Travaux Publics et des Transports, datée du $1^{\text {er }}$ décembre 1947, portant pour objet : utilisation et entretien de la base sous-marine de Saint-Nazaire, p. 2-3 : « d) l'installation d'une voie ferrée dans chacun des boxes intéressés desservant les magasins constitués [...] et assurant leur raccordement avec les voies ferrées qui bordent la façade Ouest de la Base par ouvertures des brèches nécessaires dans le béton ". Le secrétaire d'État conclut : "Ces travaux bien que modifiant d'une façon notable l'utilisation éventuelle de la Base sous-marine en temps de guerre ne paraissent pas cependant devoir le compromettre. Il paraît donc facile de réaliser un accord sur leur exécution. " 
an $)^{55}$ ». En septembre 2011, Daniel Sicard indiquait que Cargill devait " s'installer sous-peu » à Montoir-de-Bretagne et ainsi libérer les alvéoles-Nord de la base ${ }^{56}$. Ces alvéoles, sont d'une grande importance historique, car elles contiennent - ou contenaient - les espaces de couchage du personnel de la base et des sous-mariniers qui devaient partir en mission durant la Seconde Guerre mondiale. Le déplacement de l'usine correspondait à une volonté de la mairie. Toutefois le regroupement d'activités de la société à Montoir-deBretagne n'aura finalement pas lieu avant plusieurs années car " en 2012, la société américaine obtient l'autorisation de rester près de la base sousmarine jusqu'en $2035^{57}$ ". La pérennité économique de cette importante société, couplée certainement à une amélioration des techniques de trituration et à sa spécialisation dans le tournesol, a fait que la ville de SaintNazaire et ses partenaires ont modifié leurs intentions initiales.

Durant les années 1960, les alvéoles 6 à 14 auraient même également servi de port d'hivernage à des bateaux de plaisance. Toutes ces diverses formes de réinvestissement - en dehors de celles de la Défense à Brest, Lorient et La Pallice - par des entreprises liées ou non au monde maritime mettent bien en lumière les usages éclectiques qui ont pu être ceux de ces bâtiments en béton-armé. Les concessions sont accordées par les gestionnaires des anciennes bases de sous-marins et des entreprises de tout l'hexagone qui demandent d'elles-mêmes à s'installer dans ces bunkers. Les demandes d'occupation des alvéoles-bunkers sont relativement importantes pour les U-bunkers de Saint-Nazaire et de La Pallice, ainsi que pour les bunkers-écluses de ces deux villes. Une demande d'installation d'une usine de traitement de déchets d'animaux marins dans l'écluse couverte du bassin de Saint-Nazaire au cours de l'été $1958^{58}$ ou, toujours durant cette même période, l'utilisation d'un arrière-box de l'U-bunker comme entrepôt par la Société Générale des Docks Maritimes, en sont des exemples parmi tant d'autres. Le rapport de l'ingénieur subdivisionnaire du service maritime des Ponts et Chaussées, daté du 21 avril 1959 ${ }^{59}$, était d'ailleurs

55. T. H. [Thierry HAMEAu?] " 1964-2014 : 50 ans de présence à Saint-Nazaire ", OuestFrance édition La Baule - Guérande du 13 juin 2014, 345 mots.

56. Cette information a été obtenue lors d'un entretien accordé par Daniel Sicard à l'auteur en septembre 2011.

57. T.H. « 1964-2014 : 50 ans de présence à Saint-Nazaire ", cf. note ci-dessus.

58. Lettre interne de l'ingénieur d'arrondissement datée du 26 juillet 1958, à l'intention de l'ingénieur en chef de Saint-Nazaire portant pour objet : "Installation d'une usine de poudre de poisson dans l'Ecluse couverte ", Arch. dép. Loire-Atlantique. Anita Conti, " journaliste, femme de lettres [et] marin " représente pour " le compte de Monsieur Pechnard " l'entreprise. Ce dernier est un industriel " possédant déjà une très importante usine de ce genre dans la région parisienne. " (Lettre du président de la chambre de commerce Jean Laine à l'ingénieur en chef Siegfried, datée du 19 septembre 1958, Arch. dép. de Loire-Atlantique). Ces informations montrent bien que les demandes d'utilisation des U-Boot-Bunker, de la part de ces entrepreneurs ne sont pas superflues.

59. Ponts et Chaussées, rapport du subdivisionnaire daté du 21 avril 1959, portant pour objet : "Extension de la convention de la Base sous-marine ", 6 p., Arch. dép. de Loire-Atlantique. 
assez explicite sur le sujet des potentialités que pouvait offrir l'U-bunker de Saint-Nazaire :

« La Base sous-marine et les terrains annexes constituent indiscutablement un ensemble offrant des possibilités portuaires et industrielles considérables et susceptibles de connaître un développement important en liaison avec l'activité du port, si des moyens suffisants sont réunis pour permettre sa mise en valeur. "

Les moyens proviennent justement en 1959 du classement de la ville de Saint-Nazaire en " zone spéciale de conversion ${ }^{60}$ ". La ville bénéficie ainsi de certains allégements fiscaux et de primes d'équipement qui servent, entre autre, pour l'aménagement des alvéoles-bunkers. L'utilisation des alvéolesbunkers de Bordeaux et Saint-Nazaire est permise par la Marine Nationale. Mais, pour différentes raisons, la Défense se désintéresse des U-bunkers de Bordeaux et de Saint-Nazaire. Pour Saint-Nazaire, la raison militaire de l'abandon progressif de l'U-bunker au domaine civil, est due au résultat négatif d'une étude sur la capacité de résistance de la structure face à une éventuelle attaque nucléaire ${ }^{61}$. En pleine Guerre Froide, il semble logique que la Défense soit en quête d'éventuels refuges ${ }^{62}$. Cependant gérer de tels patrimoines est une lourde charge. Comme les ports de Saint-Nazaire et de Bordeaux n'ont jamais été des arsenaux militaires, la Marine ne souhaite pas consacrer un budget supplémentaire à la réutilisation des $U$-Boot-Bunker de ces deux villes. S'ajoute à cela le fait que l'accès au port de Bordeaux a été impossible durant une année entière après la Libération de la ville. Enfin, au-delà des destructions externes et internes de l'U-bunker, l'embouchure de la Gironde est située à 98 kilomètres de Bordeaux. Naviguer du golfe de Gascogne aux bassins à flot s'avère long et compliqué. Les Italiens de la BETASOM l'avaient déjà constaté durant la guerre et ils bénéficiaient, avant l'arrivée des U-Boote allemands, d'une base subsidiaire à La Pallice. Par conséquent, si la Marine Nationale demeure propriétaire et garante des lieux, elle n'utilise plus les bases de Saint-Nazaire et de Bordeaux dès les années 1960.

Mais la crise des années $1980^{63}$ porte un coup de semonce à de nombreuses activités économiques liées au monde portuaire et notamment aux industries installées dans les U-Bunker. Seule peut-être, l'entreprise Reno qui avait " adopté une stratégie entrepreneuriale ${ }^{64}$ " parvient à produire

60. Arrêté du 27 mars 1959.

61. D'après des documents consultés dans différents SHD de France, mais non déclassifiés, ces "étude[s] de la résistance des B.S.M[s] sous l'effet d'une explosion atomique " se réalisent au début de l'année 1960.

62. La base de La Pallice fait figure d'exception à ce sujet.

63. BAnTignY, Ludivine, Histoire de la France contemporaine : de 1981 à nos jours. La France à l'heure du monde, Paris, le Seuil, 2013, 512 p. Voir aussi concernant le port de Bordeaux : FERNANDEZ, Alexandre, "Sur la disparition des activités de construction navale à Bordeaux ", p. 263-273, Revue d'Histoire Maritime n 7, 2007.

64. EcK, Jean-François, Les entreprises françaises face à l'Allemagne de 1945 à la fin des années 1960, Paris, Institut de la gestion publique et du développement économique, $2003,656 \mathrm{p}$. 
en 1983 entre 80000 et 90000 tonnes d'hyperphosphates. Elle se maintient jusqu'en février 1991, date à laquelle elle cesse toute activité à SaintNazaire. L'entreprise Cargill quant à elle fait figure d'exception, comme cela a été vu précédemment. L'entreprise Shars, spécialiste en chaudronnerie et mécanique générale, qui utilisait les formes de radoub de l'U-Boot-Bunker de Bordeaux pour y réparer de petits navires, se réoriente dans les années 1970 vers les engins de travaux publics ${ }^{65}$. Les bassins à flot de l'U-bunker de Bordeaux ne semblent donc plus utilisés. Plus globalement, les deux bassins à flot de Bordeaux commencent à devenir des ports d'hivernage. Ils sont finalement déclassés par le Port Autonome en 1982. L'U-Boot-Bunker de Saint-Nazaire, redevenu un élément non productif est de nouveau perçu comme une barrière coupant la ville de son port. La période de la transition industrielle des anciennes bases de sous-marins semble terminée.

Les villes de Bordeaux et de Saint-Nazaire se retrouvent ainsi avec des édifices en béton dont elles ne savent que faire. Mais, comme en 1948, de nouvelles idées pour réinvestir ces bâtiments apparaissent alors. Développer toutes les propositions dépasserait le cadre de cet article, revenir néanmoins sur quelques exemples majeurs de la nouvelle mutation des U-Boot-Bunker de Bordeaux et Saint-Nazaire semble pertinent.

\section{La transformation des " bunkers industriels " en lieux culturels, entre concertation et concurrence}

En $1979^{66}$ la ville de Saint-Nazaire élabore un projet pour faire face à "plusieurs handicaps ${ }^{67}$ ". Globalement :

"Saint-Nazaire était identifiée et s'identifiait exclusivement à la construction navale, qui subissait une succession de crises cycliques et profondes. [...] Le regard des Nazairiens sur leur cité se heurtait alors à celui porté de l'extérieur : la ville était contournée pour accéder aux stations balnéaires et Nantes captait tout le développement tertiaire, les services, l'enseignement supérieur, la recherche ${ }^{68} \ldots$ ".

Sur l'interface portuaire, les entreprises Reno et Cargill poursuivent leurs activités au sein de l'U-bunker. "L'ancienne gare " des chemins de fer, telle qu'elle est encore dénommée par les Nazairiens et remplacée par une autre à l'est du centre-ville, demeure à l'état de ruines. Les alentours de l'U-Boot-Bunker n'invitent pas à la promenade. Saint-Nazaire, ville nouvelle reconstruite selon un plan fonctionnaliste, doit retrouver, au-delà de son attractivité originelle de port de départs et d'arrivées transatlantique, " son

65. PERIZ, Didier, Bacalan Story..., op. cit., p. 74.

66. Les conclusions de ce plan de référence sont lisibles en 1982.

67. BatTEuX, Jöel et GuEtny, Noël, Vouloir sa ville, op. cit. Expression également attribuée à THÉRY, Laurent, dans MASBOUnGI, Ariella et al., "Saint-Nazaire. Assumer sa modernité ", Projet Urbain, n $^{\circ}$ 17, juillet 1999, p. 32.

68. BATTEUX, Joël, « Fondements et stratégie urbaine de Saint-Nazaire », p. 131- 137, dans Masboungi, Ariella, Audouin, Jean et al., Estuaire Nantes - Saint-Nazaire, Écométropole mode d'emploi, Paris, Le Moniteur, sept. 2012, p. 207. 
sens " - « sa vocation portuaire ${ }^{69}$ ». L'une des trois conclusions est " d'ouvrir la ville sur le port et la mer ». Une nouvelle municipalité est élue en 1983 et Joël Batteux, ancien adjoint à l'urbanisme, remplace François Blancho. La municipalité met alors en place le Projet global de développement (PGD), lequel semble suivre une démarche sensiblement proche des Internationale Bauausstellungen (IBA) allemands ${ }^{70}$, " à la fois économique et urbain, social et culturel ${ }^{71}$ ". Le PGD est détaillé comme une IBA, en plusieurs volets, dont le premier est "Ville-Port 1 ". Cette méthode "PGD ", découpée en plusieurs étapes, permet de suivre et si besoin de modifier la réalisation de la transformation urbaine au cours des années. Ainsi le volet "Ville-Port 1 ", qui concerne en particulier l'U-bunker et ses terre-pleins, peut s'inscrire aisément dans la période charnière du changement de siècle, entre 1990 et 2002. La mise en place d'une zone d'aménagement différé (ZAD) en $1984^{72}$ facilitant l'achat des terrains attenant à l'U-bunker sur une quinzaine d'hectares ainsi que sa mise en lumières par Yann Kersalé, sont peut-être des mesures " off-Ville-Port ${ }^{73}$ ". " Ville-Port 1 " permet de faciliter les accès du centre-ville au port, en créant notamment une passerelle piétonne en béton allant du terre-plein, transformé en parking, au toit de l'U-bunker. Tout SaintNazaire peut ainsi gravir et dominer la masse de béton-armé. L'installation de l'espace muséographique sur l'histoire des paquebots, Escal'Atlantic, au sein même de l'alvéole-bunker $n^{\circ} 6^{74}$ et plus précisément sur l'ancienne darse d'où partaient avant-guerre les paquebots, fut l'élément majeur de "Ville-Port 1 ». La ville étant désormais reconnectée au port, une seconde étape semblait nécessaire : "Faire descendre le centre-ville vers le port ${ }^{75}$." "Ville-port 2 " permet donc l'installation d'un espace culturel accueillant des événements temporaires avec le Life (Lieu international des formes émergentes) et l'intégration du VIP, salle dédiée aux musiques actuelles. Ce dernier se trouvait auparavant face aux alvéoles-bunkers, de l'autre côté du bassin de Saint-Nazaire. Un projet de construction d'habitat social et privé, ainsi que l'implantation osée d'une galerie commerciale à l'emplacement de l'ancienne Maison du Peuple, forme le « Ruban bleu » entre le cœur de

69. BATtEux, Joël, "Fondements et stratégie urbaine de Saint-Nazaire ", op. cit., p. 132.

70. Un IBA est bien plus qu'une exposition d'urbanisme et d'architecture, c'est le programme structurel d'un Land. "L'IBA est dirigée par un comité de pilotage présidé par le ministre de l'Urbanisme " indique une note de la page 169 de l'ouvrage de MASBOUNGI, Ariella, Estuaire Nantes - Saint-Nazaire, op. cit. Voir aussi : LouBIÈre, Antoine et PoulizAC, Morganne, "Une invitation au voyage ", p. 29 dans " Hamburg à l'heure de l'IBA ", dossier de la revue Urbanisme ${ }^{\circ} 390$ automne 2013, p. 27-65.

71. BATTEUX, Joël, "Fondements et stratégie urbaine... ", art. cit.

72. Allaman, Martine, "Saint-Nazaire retrouve son port et sa mémoire ", p. 20-23, Diagonal n 136 , mars-avril 1999.

73. "La Nuit des Docks " de Yann KersalÉ est une idée attribuée à Joël BATTEuX, maire de Saint-Nazaire. Cette mise en lumière, phénomène de valorisation du patrimoine, commence à se développer dans quelques villes européennes à partir de la fin des années 1980.

74. Saint-Nazaire Magazine $\mathrm{n}^{\circ} 113$, février 1999, p. 9.

75. MINSSART, Sophie, « Le projet Ville-Port... ou l'histoire des relations entre la ville, son port, l'estuaire et la mer ", p. 138-147, dans MASBOUnGi Ariella et al., op. cit. 
ville et la base de sous-marins. L'étape de réalisation "Ville-Port 3 " est lancée en 2010.

Les réalisations urbanistiques de Saint-Nazaire qui commencent à la fin des années 1980, n'auraient certainement pu se concrétiser de la même manière sans la présence et l'intervention de différents acteurs. Jacques Donzelot estime en effet que " la célébration de l'année du Patrimoine en 1980, à l'initiative de Valéry Giscard d'Estaing, peut être considérée comme le moment où se trouve inauguré ce nouveau rapport au passé ${ }^{76}$ ". Mais les Nazairiens ne devaient-ils pas " [se] reconnaître [eux]-mêmes avant d'espérer [se] faire reconnaître des autres ${ }^{77}$ " ? La ville signe avec l'État une convention de développement culturel en 1982, dont l'objectif premier est " la mise en évidence de l'identité nazairienne ${ }^{78}$ ". Cette convention facilite la création et l'installation d'un écomusée, sur le modèle de celui fondé par Georges-Henri Rivière ainsi que sur celui créé en 1972 au Creusot. Avant même de s'installer entre le quartier du Petit Maroc et l'écluse-bunker, la directrice du jeune écomusée, Michèle Nolla, fait part de l'intention de la ville d'acquérir un ancien sous-marin de la Marine Nationale. Afin d'adopter les techniques de mise en valeur et de gestion d'un tel patrimoine, l'équipe responsable du projet se rend au complexe militaire de Keroman, puis à Gosport pour visiter le Royal Navy Submarine Museum. C'est finalement l'Espadon, un sous-marin déclassé de la Marine, qui rejoint le port de SaintNazaire le 23 août 1986. Une fois transformé et rendu accessible au public, il est inauguré comme sous-marin musée le 11 mai $1987^{79}$. L'écomusée est un atout pour la ville de Saint-Nazaire. Au-delà de son espace d'exposition sur l'histoire de la ville portuaire, il s'inscrit comme un véritable outil pour favoriser la compréhension du PGD auprès des habitants. C'est l'écomusée qui organise les premières visites historiques, pédestres et en bus, où guides et témoins échangent sur différentes thématiques liées à l'histoire de Saint-Nazaire. La construction de la passerelle n'est pas encore commencée que l'écomusée organise, durant les Journées du Patrimoine, des visites internes des alvéoles-bunkers et du toit de la base. Parallèlement à ces actions historiques et mémorielles, une association de Tintinophiles se crée en 1992 à Saint-Nazaire. Son objectif est simple : "Commémorer le passage de Tintin, Milou et le capitaine Haddock à Saint-Nazaire ${ }^{80}$. " Le développement et les moyens mis en œuvre sont remarquables. L'association loi 1901 "Les 7 soleils " développe des actions qui s'intègrent dans le Projet global de développement. Les deux projets ont, en effet, le même objectif

76. Donzelot, Jacques, La France des cités - Le chantier de la citoyenneté urbaine, Paris, Fayard, 2013, p. 88-89.

77. BATtEuX, Joël, Vouloir..., op. cit., p. 69, citation exacte : "Nous devions commencer par nous reconnaître nous-mêmes avant d'espérer nous faire reconnaître des autres. "

78. Ibid.

79. G. RAMSEY, Winston et al., " Preservation. Saint-Nazaire Ecomuseum », p. 24-25, dans "The Raid on Saint-Nazaire", ATB, n 59, London, 1988.

80. Chemin, Jean-Claude, «Tintin », p. 70-71, Place Publique, Nantes, janvier-février 2009. 
global : celui de re-valoriser Saint-Nazaire. Ainsi de $1995^{81}$ à nos jours, un parcours de vignettes agrandies est installé. Celles-ci jalonnent la ville de la nouvelle gare à l'U-Boot-Bunker. Enfin, pour mener à bien le PGD, la ville crée en 1989, à partir d'un comité issu de la direction de l'Aménagement, une association constituée

" [d']une équipe d'ingénierie chargée de l'élaboration et du suivi du projet global de développement. " "Elle ne ressemble à aucune structure connue. [...] Composée de vingt personnes (ingénieur, architectes, paysagistes, urbanistes, économistes...), elle intervient principalement dans les domaines du développement urbain, économique et social. [...] La DDRN fonctionne de façon transversale. Elle ne prend pas de décision tâche qui revient aux élus - mais sert d'outil d'aide à la décision $[\ldots]^{82}$."

Ainsi, comme l'avait fait l'équipe de l'écomusée en allant rencontrer des spécialistes notamment à Keroman, l'association pour le développement de la région nazairienne (DDRN) se rend à Bordeaux pour découvrir la mise en place du Conservatoire international de Plaisance (CIPB), au cours du mois de juillet 1992 .

La ville de Bordeaux avait retenu le projet de l'architecte Antoine Grumbach pour la création d'un quartier polyfonctionnel autour des bassins à flot, incluant la dimension patrimoniale des lieux ${ }^{83}$. Ce projet, qui devait être réalisé en deux phases, avec une première tranche qui aurait dû être achevée en 2006, est officiellement abandonné en 2008. La zone d'aménagement concerté (ZAC) est remplacée en 2009 par un programme d'aménagement d'ensemble (PAE) dans le cadre de "Bordeaux 2030 ». Les méthodes sont de natures différentes. Le PAE, contrairement à la ZAC, permet aux promoteurs d'acheter directement les terrains et de payer des taxes sur la réalisation des équipements de voiries. De même, plusieurs initiatives qui ont pour objectif la réutilisation de l'U-bunker sont menées parallèlement, mais elles manquent probablement de coordination. Le CIPB, dont l'objectif est « la conservation et la mise en valeur du patrimoine de la plaisance ${ }^{84}$ ", est constitué en entreprise ${ }^{85}$ et reçoit une délégation de service public le 5 novembre 1992. Il est repris sous la forme d'une "régie directe par la ville en juin $1993^{86}$ ". Il ouvre au public en juillet 1993, mais se trouve contraint à la fermeture à la fin de l'année 1997. Malgré les bons

81. Date de l'inauguration du premier agrandissement sur métal émaillé d'une des vignettes extraite de l'album de Rémi, Georges, Les 7 Boules de Cristal, op. cit.

82. AlLAMAN, Martine, "La DDRN une spécificité locale ", p. 22, dans "Saint-Nazaire retrouve..." ", art. cit. Cette structure a évolué en ADDRN : agence pour le développement durable de la région nazairienne.

83. Grumbach, Antoine \& Associés, Communauté Urbaine de Bordeaux, Projet d'aménagement du secteur des Bassins à flot de Bordeaux-Nord, juin 1998, 43 p. (Archives AURBA).

84. KLAUTH, Sophie, La difficile genèse d'un équipement public culturel, rapport de stage, Institut d'Études Politique de Bordeaux, septembre 1994, 38 p.

85. En société mixte d'économie locale (SMEL), intégrant un capital apporté par la ville de Bordeaux.

86. KLAUTH, Sophie, La difficile genèse d'un équipement public culturel, op. cit., p. 1. 
souvenirs qu'en ont les Bordelais ${ }^{87}$, le CIPB accuse un manque de recettes dû à une fréquentation fluctuante et limitée. En juin 1995, le départ de Jacques Chaban-Delmas et l'arrivée d'Alain Juppé à la mairie de Bordeaux entraînent une modification de la politique muséale de la ville et du soutien accordé au CIPB. Enfin son emplacement géographique situé au sein d'un quartier en marge, son manque d'insertion dans l'offre culturelle de la ville et le non renouvellement des collections n'ont pas facilité son maintien. Cependant, la rencontre entre la délégation nazairienne de la DDRN et l'équipe du CIPB en $1992^{88}$ a été très utile pour la ville de Saint-Nazaire. Elle réutilise ainsi l'idée des caissons étanches pouvant être installés au sein des U-bunkers. Ces caissons permettent de créer des salles à l'intérieur des U-bunkers, sans entreprendre de lourds travaux d'isolation. Le CIPB facilite également l'installation de l'association à caractère historique "Betasom " dans un des inter-boxes de la base. Cependant à partir de la fermeture de l'espace muséographique "l'association Betasom " de Bordeaux ${ }^{89}$ semble également cesser ses activités à la fin des années 1990. L'ancienne base d'U-Boote de Bordeaux continue malgré tout à attirer du public dans le cadre, entre-autres, de concerts de rock. Elle sert également d'atelier au rockeur et sculpteur de métaux, Jean-François Buisson. Face à un tel attrait pour différentes formes de cultures émergentes se développant au sein de l'U-bunker, la ville décide de le réinvestir, d'entreprendre quelques travaux à partir de la fin de l'année $1998^{90}$ et d'ouvrir un espace culturel. Ce lieu dénommé " base sous-marine " accueille des expositions et des évènements temporaires depuis le début du nouveau siècle.

Si les projets de réhabilitations et de transformations urbaines du "Bordeaux Maritime " et de Saint-Nazaire " ville-port " sont désormais en cours, voire bien avancés, l'histoire des U-Boot-Bunker de ces deux villes semble cependant oubliée. C'est grâce à la transmission de l'histoire des lieux, par l'écomusée de Saint-Nazaire et par les associations bordelaises ${ }^{91}$ que les prémices des réalisations urbaines autour des U-bunkers furent réalisées. Cette histoire ne servirait-elle donc qu'à la communication des grands projets?

87. Au dire des témoignages des participants aux visites historiques organisées par l'association Mers \& Océans.

88. Rapport interne de Martine Staebler au directeur de l'écomusée de Saint-Nazaire Daniel Sicard, daté du 29 juillet 1992 et portant pour objet : " Saint-Nazaire ville croisière ", 6 p. (Écomusée de Saint-Nazaire).

89. Le président de cette association fut l'auteur du premier mémoire universitaire sur les bases de sous-marins de Bordeaux, cf. VIDALENC, Pierre-Laurent, La Base sous-marine de Bordeaux, lieu de mémoire, lieu d'avenir, certificat international d'écologie humaine, Talence - Bordeaux 1, 18 mai 1998.

90. Dossier Base-sous marine, archives municipales de Bordeaux.

91. En septembre 2012 l'auteur avec quelques soutiens a créé l'Association Mers \& Océans, dont l'objectif est la promotion des fortifications situées à proximité d'une mer ou d'un océan. Celle-ci organise régulièrement des visites à caractère historique de l'ancienne base italienne de Bordeaux et de l'U-bunker allemand ainsi que des visites spécifiques. L'Association Mers et Océans envisage également de faire connaître l'histoire de la base de La Pallice. 
Les villes de Bordeaux et de Saint-Nazaire ${ }^{92}$ ont eu à subir la construction des U-Boot-Bunker sur leur espace urbain. Après avoir songé à la destruction de ces vestiges de la Seconde Guerre mondiale, les ports, les chambres de commerce et d'industrie, les municipalités et la Marine Nationale ont constaté que ceux-ci pourraient avoir une utilité pour l'implantation d'activités industrielles. De garages à submersibles ennemis, les U-bunkers deviennent ainsi des hangars industriels en béton armé au service de l'économie française. Les industries profitant de ces bâtiments ne sont pas toutes respectueuses de l'environnement et les conditions de travail peuvent en pâtir, cependant l'exemple de l'adaptabilité de Cargill qui poursuit son activité à Saint-Nazaire semble plutôt positif. Les crises économiques sectorielles, l'évolution géopolitique générale - les matières premières ou semi-transformées importées à l'U-bunker de Saint-Nazaire étaient en majeure parties issues d'Afrique du Nord -, l'innovation technique et la modification des besoins font que ces industries périclitent à partir de la fin des années 1970 et finissent par fermer au début des années 1990. En outre, les U-bunkers ne semblent pas adaptés à l'installation d'entreprises tertiaires car, contrairement à certains blockhaus, ils sont trop imprégnés d'humidité. Or le tourisme urbain est désormais vu comme un moyen de capter des capitaux. Ceci est conjugué à la volonté d'améliorer l'espace urbain, coextensive à une démarche de réappropriation de la ville par ses habitants. Pour répondre aux aspirations de ces derniers et attirer les touristes, différentes opérations culturelles sont, dès lors, mises en place par les collectivités locales telles que les biennales d'arts, les festivals et autres grands événements (artistiques, nautiques, etc.). Ces manifestations culturelles sont rassembleuses et se tiennent au sein ou à proximité des U-bunkers. Les bâtiments qui protégeaient et cachaient des submersibles, deviennent ainsi des points centraux visibles, des paysages mis en avant par les villes. Si l'aspect loisir semble l'avoir emporté sur l'aspect historique de ces lieux, les U-bunkers demeurent néanmoins des éléments patrimoniaux des cités portuaires. Mais la transmission des questions de mémoire et de l'histoire de ces édifices peut-elle être uniquement proposée sous la forme de visites-guidées temporaires? Enfin, la diversité des projets et des utilisations civiles déjà mises en œuvres pour la réutilisation des U-Bunkers, pourrait favoriser de nouvelles réflexions collectives et innovantes quant au devenir de celui de La Pallice et prochainement celui de Brest ${ }^{93}$.

92. L'auteur tient à remercier l'équipe des archives municipales de la ville de SaintNazaire pour l'aide apportée à la recherche. Il remercie également madame la documentaliste de l'agence de l'urbanisme de Bordeaux-Aquitaine, le personnel du Grand Port Maritime de Bordeaux, le département urbanisme de la Communauté Urbaine de Bordeaux et l'équipe de la base sous-marine de Bordeaux.

93. L'U-bunker de Brest est moins utilisé par la Marine Nationale, aussi il apparaît possible qu'il soit remis au domaine civil. 
Figure 1 - L'U-bunker de Bordeaux en février 2012. En arrière-plan, un vestige nautique du Centre international de la Plaisance de Bordeaux (DR).

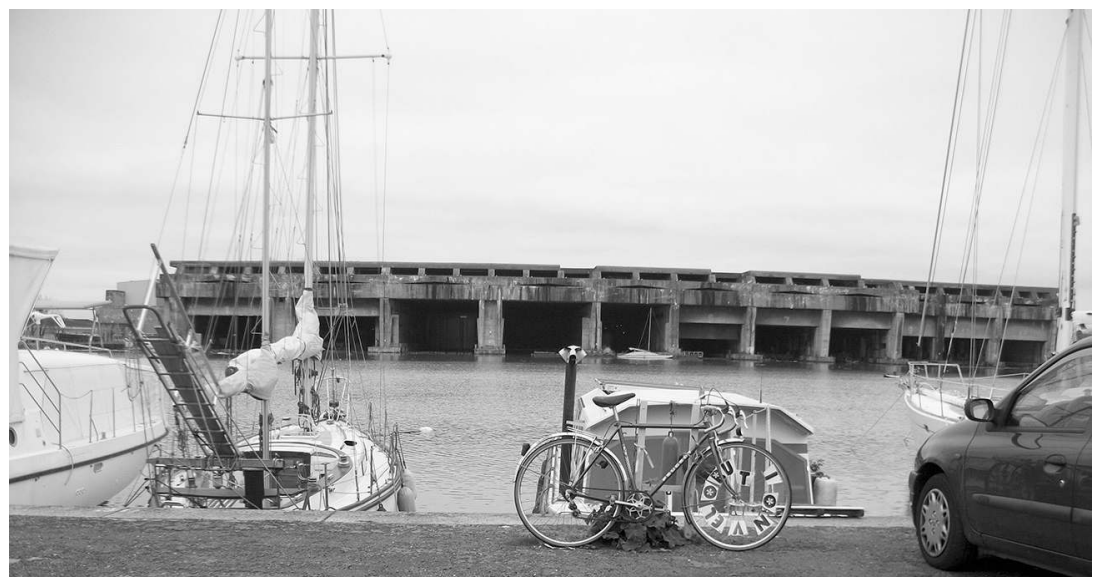

Figure 2 - L'U-bunker de Saint-Nazaire avec les deux grues sur le quai du Commerce en septembre 2011 (DR)

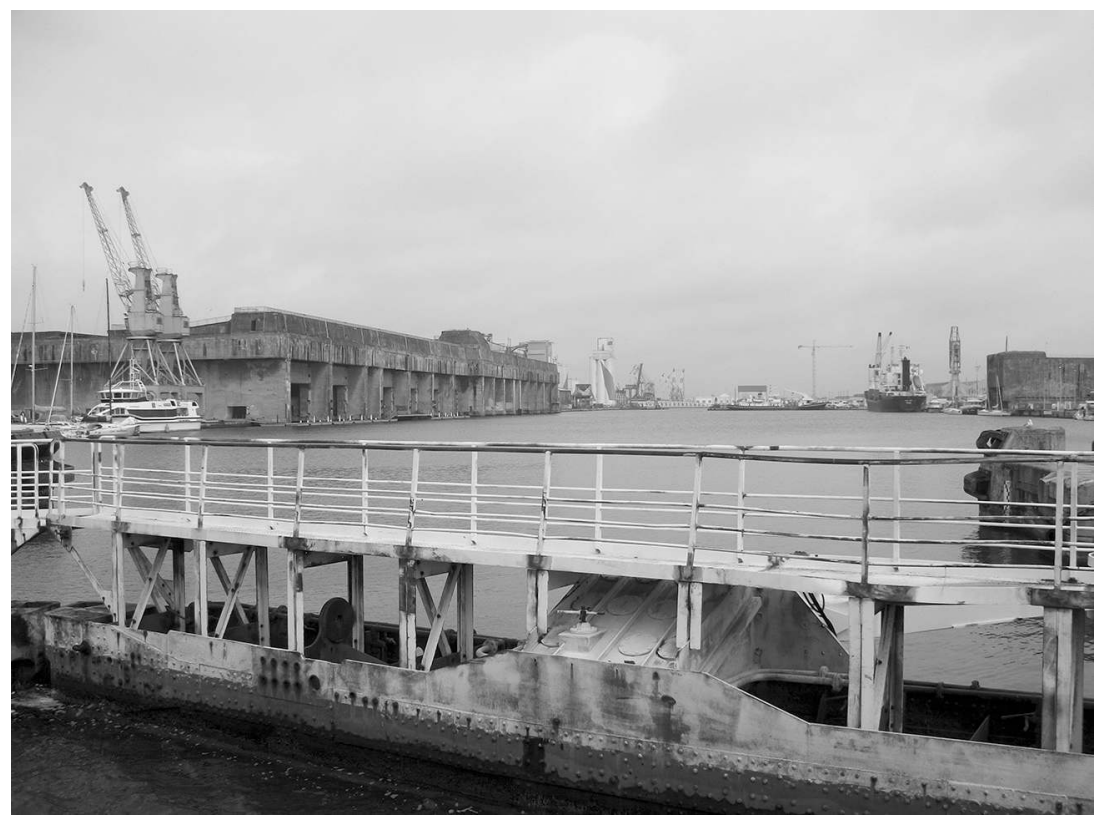


La délicate gestion des U-Boot-Bunker à Bordeaux et Saint-Nazaire

Figure 3 - Les U-bunkers construits par le Reich (1940-1945) (J.-B. Blain, P. Brunello)

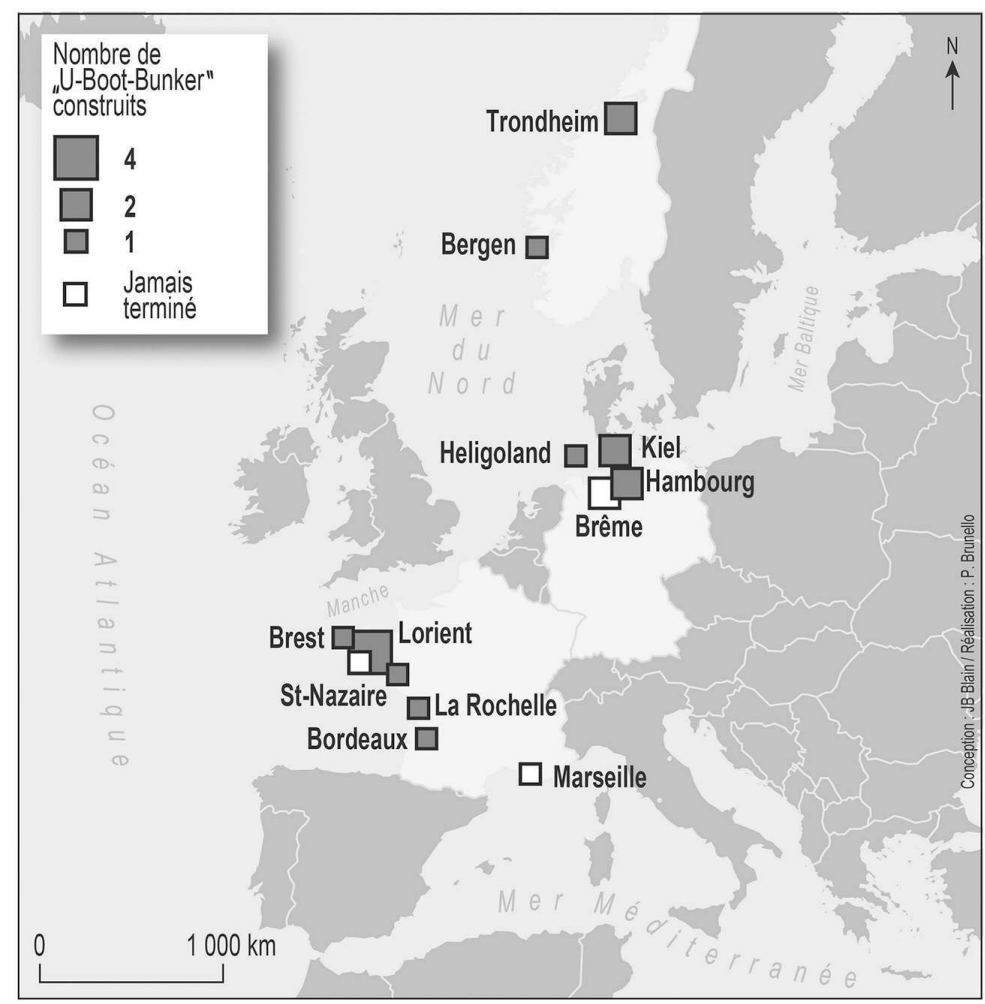

Figure 4 - L'écluse-bunker de Bordeaux en 1946 ou 1947 avant sa destruction (Port maritime de Bordeaux)

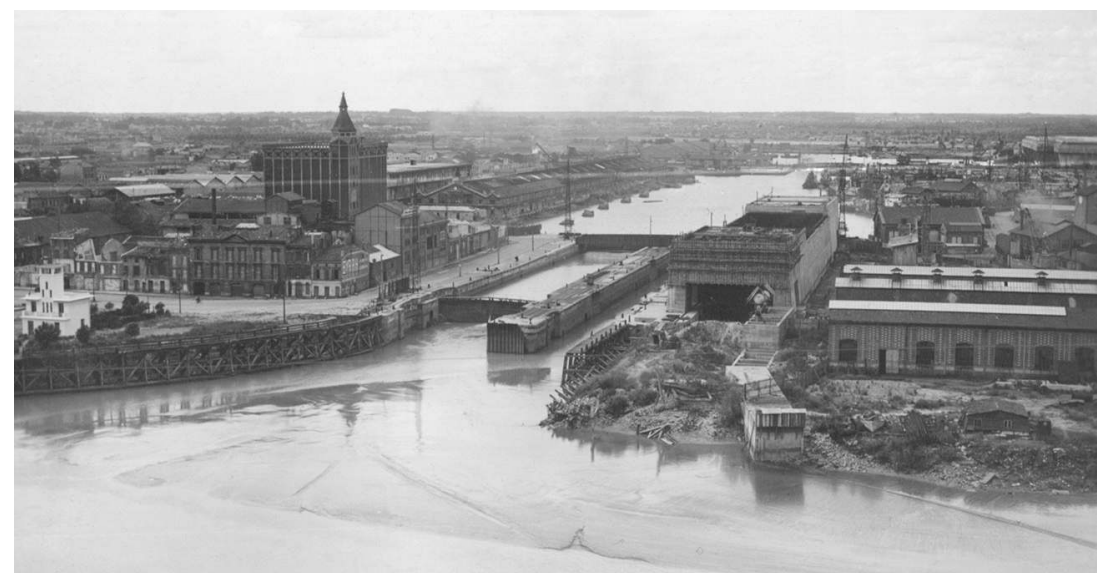


Jean-Baptiste BLAIN

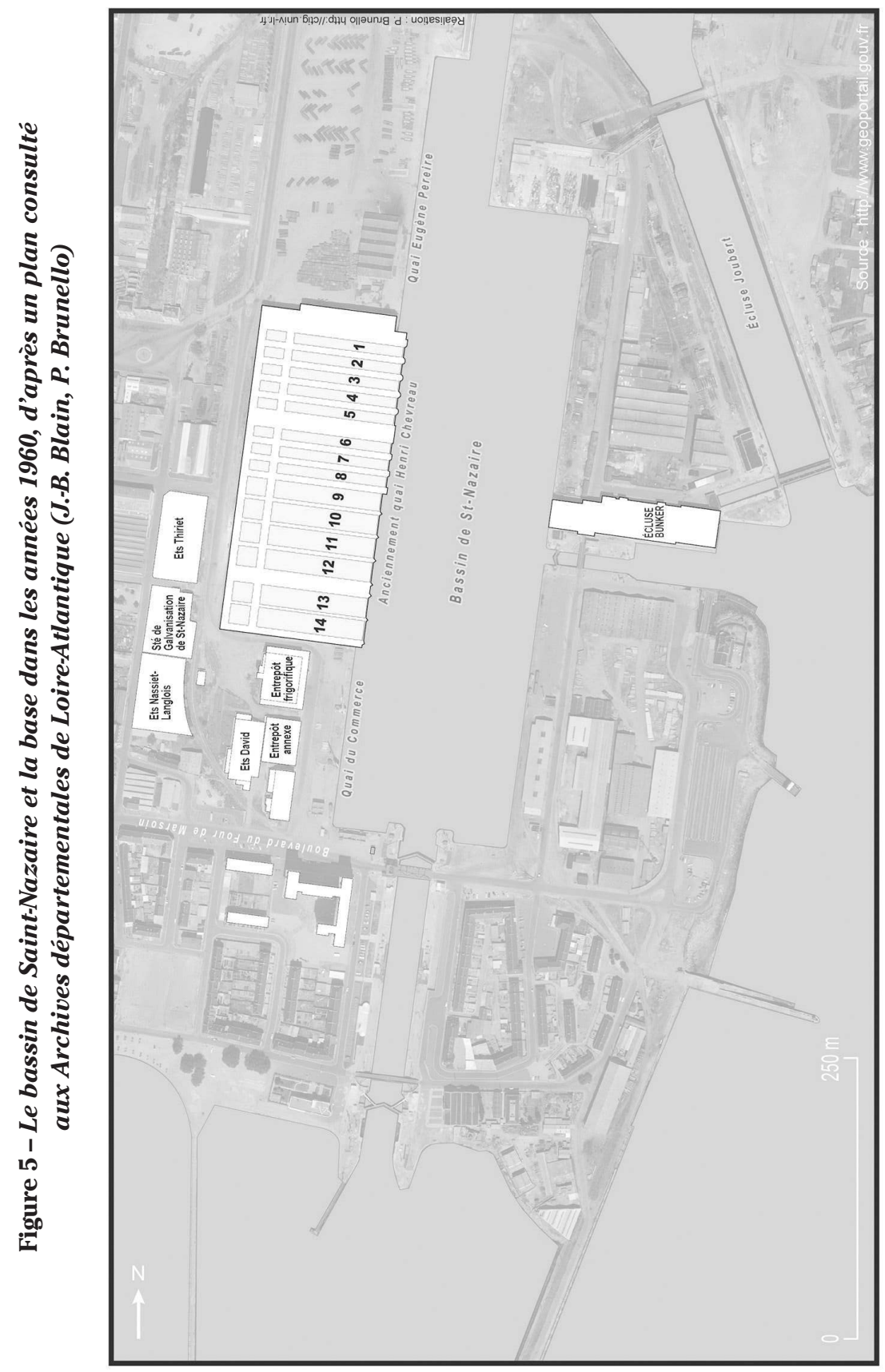




\section{RÉSUMÉ}

Édifiés durant l'Occupation nazie les $U$-Boot-Bunker sont les rares témoins architecturaux de cette période sombre. Les U-bunkers construits sur la façade atlantique française, n'ont jamais subi de tentative de destructions. Au contraire, après la guerre ces immenses bunkers ont différentes fonctions. Certains, dont les anciens bunkers à submersibles de Bordeaux et SaintNazaire, ont rapidement été mis à disposition des ports pour y accueillir des entreprises industrielles. Cependant, à la fin des années 1980, nombre de ces entreprises ferment et les villes portuaires doivent alors intégrer " les bases sous-marines " dans leur espace urbain. Différentes méthodes sont dès lors mises en ouvre pour que les U-Boot-Bunker ne soient plus considérés comme des friches militaro-industrielles, mais comme des éléments attractifs.

\section{ABSTRACT}

Built during the Nazi occupation of France, U-boat bunkers are rare architectural witnesses of one of European history darkest periods. The U-bunkers edified on the French Atlantic coast were not destroyed by the authorities. On the contrary, these monumental bunkers were reused during the post-war period. In the case of those in Saint-Nazaire and Bordeaux, they were used by the ports as storage facilities for local industry. However, by the end of the 1980s many of the businesses had closed and cities were left wondering how they could use the old "submarine pens". New town planning was necessary to ensure that the U-bunkers would no longer be considered as military and industrial wastelands, but instead be redeveloped as attractive structures. 